\title{
Costs of Coexisting Depression and Diabetes
}

\author{
Hillary R. Bogner, MD, MSCE ${ }^{1,2}$ and Heather F. de Vries McClintock, MSPH, MSW, PhD ${ }^{1,2}$ \\ 'Department of Family Medicine and Community Health, University of Pennsylvania, Philadelphia, PA, USA; ${ }^{2}$ Center for Clinical Epidemiology and \\ Biostatistics, University of Pennsylvania, Philadelphia, PA, USA.
}

J Gen Intern Med 31(6):594-5

DOI: $10.1007 / \mathrm{s} 11606-016-3675-5$

(c) Society of General Internal Medicine 2016

$\mathrm{A}$ ccording to the Global Burden of Disease Study 2010 and the World Health Organization, diabetes and depression are leading causes of disability. In the United States, approximately 25.6 million adults have diabetes mellitus (11.3\% of the population). The burden of diabetes is anticipated to grow, with estimates suggesting that the proportion of the population affected by diabetes and related costs will at least double in the next 25 years, yet the proportion of adults whose diabetes is controlled is decreasing over time and is particularly low among older adults and ethnic minorities. Depression is a risk factor for diabetes and risk of depression is increased by a factor of two in patients with diabetes. Depressive symptoms contribute to poor patient engagement, poor adherence to medication and dietary regimens, poor glycemic control, reduced quality of life, and increased health expenditures. ${ }^{1}$

The influence of depression on outcomes for diabetes includes both biological mechanisms such as increased inflammation, poor self-care, and decreased adherence to treatment regimens. For patients with diabetes, depression has been specifically linked to prognostic variables such as microvascular and macrovascular complications. ${ }^{2}$ Depression has been found to increase all-cause mortality even in the context of good glucose control. ${ }^{3}$ For diabetes, increasing attention has been given to the principle that day-to-day management should be in the hands of patients themselves. Self-care activities (e.g., checking one's own finger-stick blood sugar), health behaviors (e.g., smoking, alcohol use, diet, physical activity), and patient choice (e.g., whether to start a new medication or undergo a procedure) all profoundly affect outcomes in diabetes and all are strongly influenced by depression. The biological, social, psychological, and behavioral links between depression and diabetes provide a strong incentive to examine the healthcare costs of depression in adults with diabetes. Moreover, national estimates of the healthcare costs of comorbid depression and diabetes are essential for providing decision makers with needed information to inform policy and practice changes.

In this issue of JGIM, Egede et al. ${ }^{4}$ investigate the trends in healthcare costs over 8 years in patients aged 18 years and

Published online March 23, 2016 older with diabetes. Based on International Classification of Diseases, Clinical Modification (ICD-9-CM) codes to measure the presence or absence of a depression diagnosis and the two-item Patient Health Questionnaire (PHQ-2) to measure the presence or absence of depressive symptoms, patients with diabetes were classified as no depression, unrecognized depression, asymptomatic depression, or symptomatic depression. The authors report that patients with unrecognized and asymptomatic depression had healthcare expenditures that were \$2000-\$3000 higher compared to patients with no depression, and patients with symptomatic depression had healthcare expenditures that were $\$ 5000$ higher compared to patients with no depression after adjustment for potentially influential covariates. The authors adjusted for sociodemographic factors, comorbidities, and time trend covariates. No depression was associated with the lowest total, inpatient, outpatient, prescription, office-based, and emergency room costs, and symptomatic depression was associated with the highest costs of all types. The authors found the higher medical expenditures associated with depression persisted over time.

This study used data from the 2004-2011 Medical Expenditure Panel Survey (MEPS), a nationally representative estimate of healthcare expenditures maintained and cosponsored by the Agency for Healthcare Research and Quality (AHRQ). The MEPS data spanned 8 years and involved 15,548 adults with diabetes. The large ethnically diverse and nationally representative sample and the pooled data set over 8 years are significant strengths. Because of the focus on costs due to medical expenditures, other nonmedical costs related to caregiving, community services, short-term sick leave, and social services are not included and are an important area of future inquiry. The overall mean medical expenditures for patients with diabetes and no depression was \$10,016 (95 \% CI 9589$10,442)$, with unrecognized depression was $\$ 15,155$ (95\% CI $13,587-16,723)$, with asymptomatic depression was $\$ 16,134$ (95\% CI 14,885-17,382) and with symptomatic depression was $\$ 20,105$ (95\% CI 18,103-22,106). Pairwise comparison tests showed the mean expenditure between all groups were significantly different, except between asymptomatic and unrecognized depression. However, compared to unrecognized depression, the costs associated with asymptomatic depression were higher for prescriptions and office based visits, presumably as a result of treatment. The benefit of treatment is consistent with the lower overall mean medical expenditures 
for patients with asymptomatic depression compared to symptomatic depression, and is also supported by previous work on the cost-effectiveness of interventions to improve depression care.

Unfortunately, depression is often unrecognized and untreated. The US Preventive Services Task Force (USPSTF) recently renewed attention to the problem of unrecognized and untreated depression and updated the 2009 USPSTF recommendation on screening for depression in adults. The USPSTF now recommends screening for depression in the general adult population, and that screening is implemented with adequate systems in place to ensure accurate diagnosis, effective treatment, and appropriate follow-up. Direct evidence indicates that screening may reduce the prevalence of depression in a specific population, particularly when additional support for treatment is available (e.g., treatment guidelines, care management, and availability of trained depression care providers). ${ }^{5}$ The USPSTF does not consider the costs of providing a service in this assessment, but notes that persons with chronic illnesses, such as diabetes, are at increased risk of developing depression. Effective care for depression in patients with diabetes is challenging. Mental health services are often separated from primary care leading to poor access to psychological services and other effective treatments.

Integrated care (e.g., co-locating mental health providers in primary care settings coupled with care management) has been shown to help improve clinical outcomes and reduce costs. For example, Improving Mood-Promoting Access to Collaborative Treatment (IMPACT), a collaborative care program that involved a nurse or psychologist working in the primary care office to support the care from the primary care physician, was associated with improvements in depressive symptoms, functioning, and quality of life. ${ }^{6}$ Katon and colleagues reported that patients with depression and poorly controlled diabetes or cardiovascular disease in practices with collaborative care managed by a medically supervised nurse had greater improvement in glycated hemoglobin, lipids, blood pressure, and depression, as well as better quality of life, compared to patients in usual care. ${ }^{7}$ In the Prevention of Suicide in Primary Care Elderly: Collaborative Trial (PROSPECT), persons with diabetes and depression were half as likely to die over a 5-year follow-up in intervention practices compared to usual care. ${ }^{8}$ In addition, a simple, brief integrated intervention carried out by Licensed Practical Nurses was found to improve medication adherence, glycemic control, blood pressure and/or depressive symptoms over 12 weeks of follow-up among a diverse range of patients. ${ }^{9}$ A recent systematic review of economic evidence found that integrated interventions for depression and diabetes significantly reduced depression and were cost-effective compared with usual care. In this work, two studies reported costs per Quality Adjusted Life Year (QALY) gained of \$267 to $\$ 4317$, and two studies reported the intervention dominated usual care, with net savings of $\$ 440$ to $\$ 612$ and net gains in QALYs. ${ }^{10}$

There is an urgency to finding evidence-based solutions to reduce the burden of diabetes and coexisting depressive symptoms. The Patient Protection and Affordable Care Act signed into law in March 2010 provides a new impetus for dealing with diabetes and depression. Highlighted was the need for improved communication between mental health and primary care, the inclusion of mental health management in the medical home model for diabetes and other conditions, and new models of integrated health care delivery. Integrated care is a promising development for feasibly and effectively managing complex patients with comorbid conditions like diabetes and depression. Such interventions can be carried out by nonspecialists and by nonphysician health care workers. Most adults with depression never seek mental health treatment in either primary care or mental health settings. The challenge is to develop ways to recognize vulnerable adults with depression and diabetes who may have poor access to care or who may not make optimal use of the medical system. Doing so will require that we continue to study how to integrate medical care and care for depression.

Corresponding Author: Hillary R. Bogner, MD, MSCE; Department of Family Medicine and Community Health, University of Pennsylvania, 2 Gates, 3400 Spruce St, Philadelphia, PA 19104, USA (e-mail: hillary.bogner@uphs.upenn.edu).

\section{REFERENCES}

1. Pignone M. Organized care for depression improves outcomes and reduces costs. Clin Diabetes. 2008;26(1):15-6.

2. de Groot M, Anderson R, Freedland KE, Clouse RE, Lustman PJ. Association of depression and diabetes complications: a meta-analysis. Psychosom Med. 2001;63(4):619-30.

3. Sullivan MD, O'Connor P, Feeney $\mathbf{P}$, Hire D, Simmons DL, Raisch DW, et al. Depression predicts all-cause mortality: epidemiological evaluation from the ACCORD HRQL substudy. Diabetes Care. 2012;35(8):1708-15.

4. Egede L, Walker R, Bishu K, Dismuke C. Trends in costs of depression in adults with diabetes in the United States: Medical Expenditure Panel Survey, 2004-2011. J Gen Intern Med. 2016. doi:10.1007/s11606-016-3650-1.

5. Siu AL, Force USPST, Bibbins-Domingo K, Grossman DC, Baumann LC, Davidson KW, et al. Screening for depression in adults: US preventive services task force recommendation statement. JAMA. 2016;315(4):380-7.

6. Katon W, Russo J, Von Korff M, Lin E, Simon G, Bush T, et al. Longterm effects of a collaborative care intervention in persistently depressed primary care patients. J Gen Intern Med. 2002;17(10):741-8.

7. Katon WJ, Lin EH, Von Korff M, Ciechanowski P, Ludman EJ, Young B, et al. Collaborative care for patients with depression and chronic illnesses. N Engl J Med. 2010;363(27):2611-20.

8. Bogner HR, Morales KH, Post EP, Bruce ML. Diabetes, depression, and death: a randomized controlled trial of a depression treatment program for older adults based in primary care (PROSPECT). Diabetes Care. 2007;30(12):3005-10.

9. Bogner HR, de Vries HF, Kaye EM, Morales KH. Pilot trial of a licensed practical nurse intervention for hypertension and depression. Fam Med. 2013;45(5):323-9.

10. Jeeva F, Dickens C, Coventry $\mathbf{P}$, Bundy C, Davies $\mathbf{L}$. Is treatment of depression cost-effective in people with diabetes? A systematic review of the economic evidence. Int J Technol Assess Health Care. 2013;29(4):384-91. 